Supplement of Hydrol. Earth Syst. Sci., 22, 2311-2341, 2018

https://doi.org/10.5194/hess-22-2311-2018-supplement

(c) Author(s) 2018. This work is distributed under

the Creative Commons Attribution 4.0 License.

(c) (1)
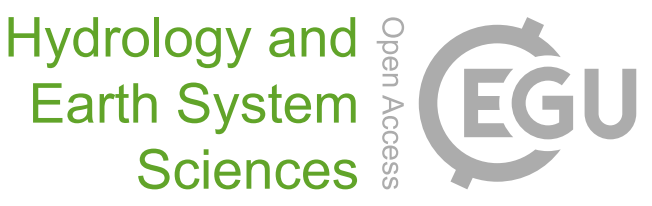

Supplement of

\title{
Regional evapotranspiration from an image-based implementation of the Surface Temperature Initiated Closure (STIC1.2) model and its validation across an aridity gradient in the conterminous US
}

Nishan Bhattarai et al.

Correspondence to: Nishan Bhattarai (nbhattar@umich.edu)

The copyright of individual parts of the supplement might differ from the CC BY 4.0 License. 

best overall performance statistics among the three models.

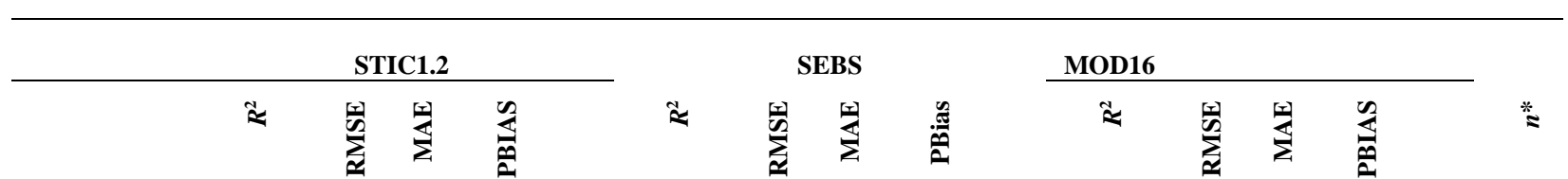

\begin{tabular}{lrrrrrrrrrrrrr}
\hline US-Me2 & $\mathbf{0 . 6 4}$ & $\mathbf{6 . 6}$ & $\mathbf{5}$ & $\mathbf{- 1 9}$ & 0.64 & 9 & 7 & 33 & 0.43 & 8.1 & 6 & -21 & 80 \\
US-Ton & 0.17 & 9.4 & 7 & 80 & 0.16 & 16.2 & 13 & 179 & $\mathbf{0 . 7 2}$ & $\mathbf{3 . 7}$ & $\mathbf{3}$ & $\mathbf{1 0}$ & 109 \\
US-SRM & $\mathbf{0 . 5}$ & $\mathbf{5 . 8}$ & $\mathbf{4}$ & $\mathbf{2}$ & 0.6 & 7.9 & 7 & 64 & 0.36 & 9.5 & 6 & -71 & 82 \\
US-SRG & $\mathbf{0 . 4 5}$ & $\mathbf{7 . 4}$ & $\mathbf{6}$ & $\mathbf{2}$ & 0.5 & 10 & 8 & 69 & 0.52 & 10.7 & 7 & -68 & 85 \\
US-Wkg & $\mathbf{0 . 3 9}$ & $\mathbf{6 . 3}$ & $\mathbf{5}$ & $\mathbf{1 6}$ & 0.4 & 12.1 & 10 & 119 & 0.53 & 8 & 5 & -64 & 128 \\
US-NR1 & 0.72 & 5.5 & 4 & -14 & $\mathbf{0 . 7 6}$ & $\mathbf{5 . 5}$ & $\mathbf{4}$ & $\mathbf{5}$ & 0.76 & 8.4 & 6 & -44 & 116 \\
US-Kon & 0.82 & 8.6 & 6 & -23 & $\mathbf{0 . 7 2}$ & $\mathbf{8 . 9}$ & $\mathbf{6}$ & $\mathbf{0}$ & 0.87 & 8.2 & 6 & -21 & 59 \\
US-KFS & $\mathbf{0 . 8 1}$ & $\mathbf{7 . 1}$ & $\mathbf{5}$ & $\mathbf{- 1 3}$ & 0.76 & 7.5 & 5 & 2 & 0.77 & 8.5 & 6 & -20 & 88 \\
US-ARM & $\mathbf{0 . 3 8}$ & $\mathbf{1 0}$ & $\mathbf{7}$ & $\mathbf{1 1}$ & 0.45 & 12.2 & 9 & 48 & 0.43 & 10 & 7 & -41 & 61 \\
US-Ne1 & 0.79 & 10.1 & 7 & -32 & $\mathbf{0 . 8 2}$ & $\mathbf{7 . 6}$ & $\mathbf{6}$ & $\mathbf{- 1 5}$ & 0.61 & 16 & 11 & -50 & 114 \\
US-MMS & $\mathbf{0 . 8 9}$ & $\mathbf{5 . 2}$ & $\mathbf{4}$ & $\mathbf{1 1}$ & 0.7 & 8.3 & 6 & 17 & 0.89 & 5.6 & 5 & 12 & 108 \\
US-NC1 & 0.75 & 7.4 & 6 & 16 & 0.65 & 8.9 & 6 & 23 & $\mathbf{0 . 7 7}$ & $\mathbf{6}$ & $\mathbf{4}$ & $\mathbf{7}$ & 75 \\
US-NC2 & 0.88 & 6.1 & 5 & -17 & 0.78 & 7.8 & 6 & -20 & $\mathbf{0 . 8 6}$ & $\mathbf{5}$ & $\mathbf{4}$ & $\mathbf{- 6}$ & 65 \\
\hline
\end{tabular}

$*_{n} n$ represents the 8-day MODIS periods when remotely sensed data, EC flux, and MOD16 data were available 
Table S2. Comparison of 8-day average daytime meteorological and radiative inputs vs. instantaneous inputs to assess how representative the 8-day average values were of each day within the 8-day period.

\begin{tabular}{lllll}
\hline Variable & $R^{2}$ & RMSE & MAE & PBIAS (\%) \\
\hline$T_{\mathrm{R}}(\mathrm{K})$ & 0.92 & 3.53 & 2.74 & 0.1 \\
$T_{\mathrm{A}}(\mathrm{K})$ & 0.900 & 3.04 & 2.34 & 0 \\
$T_{\mathrm{R}}-T_{\mathrm{A}}(\mathrm{K})$ & 0.80 & 3.16 & 2.42 & 3.8 \\
$\mathrm{RH}(\%)$ & 0.78 & 10 & 8 & 6 \\
Wind speed $\left(\mathrm{m} \mathrm{s}^{-1}\right)$ & 0.36 & 1.61 & 1.19 & 2 \\
Incoming shortwave & 0.82 & 69 & 49 & $-5 \%$ \\
radiation $\left(\mathrm{W} \mathrm{m} \mathrm{m}^{-2}\right)$ & & & & \\
\hline
\end{tabular}

Table S3. Evaluation of instantaneous ET and 8-day cumulative ET (Table 3, Page 28) from STIC1.2 and SEBS against observed ET from thirteen core AmeriFlux sites in the US combining data from one dry, one wet, and one normal year. Note: the 8-day ET estimates are derived from 8-day MODIS products (MOD11A2, MOD09A2, etc.) and 8-day average weather data (both at the satellite overview time and the 8-day average values).

\begin{tabular}{lllccc}
\hline Scales & Model & $R^{2}$ & $\begin{array}{c}\text { RMSE } \\
\left(\mathrm{mm} \mathrm{hr}^{-1} \text { or mm 8-day }{ }^{-1}\right)\end{array}$ & $\begin{array}{l}\text { MAE (mm) } \\
\left(\mathrm{mm} \mathrm{hr}^{-1} \text { or mm 8-day }{ }^{-1}\right)\end{array}$ & PBIAS (\%) \\
\hline \multirow{2}{*}{ Instantaneous } & STIC1.2 & 0.61 & 0.12 & 0.09 & -5 \\
& SEBS & 0.53 & 0.14 & 0.10 & 17 \\
\hline \multirow{2}{*}{ 8-day } & STIC1.2 & 0.66 & 7.5 & 5.4 & -3 \\
& SEBS & 0.53 & 9.8 & 7.3 & 28 \\
\hline
\end{tabular}


a)

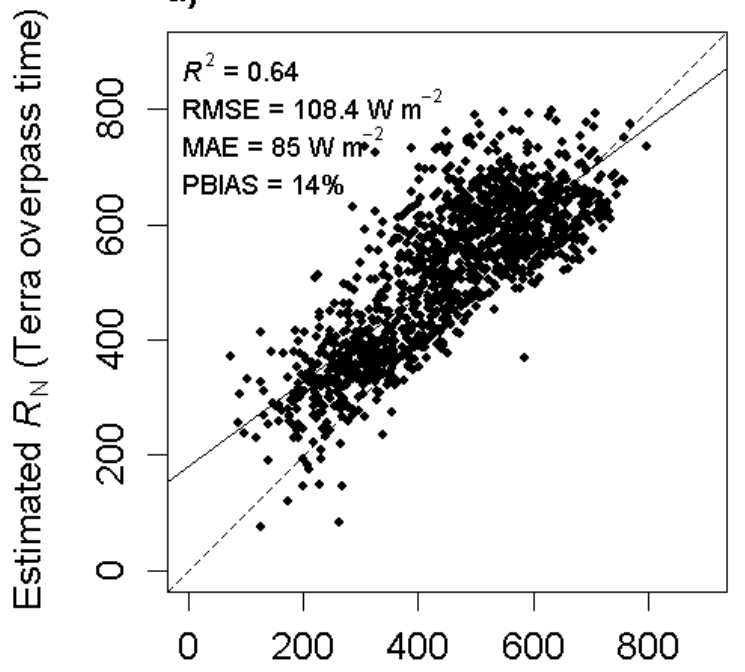

Observed $R_{N}$ (Terra overpass time) b)

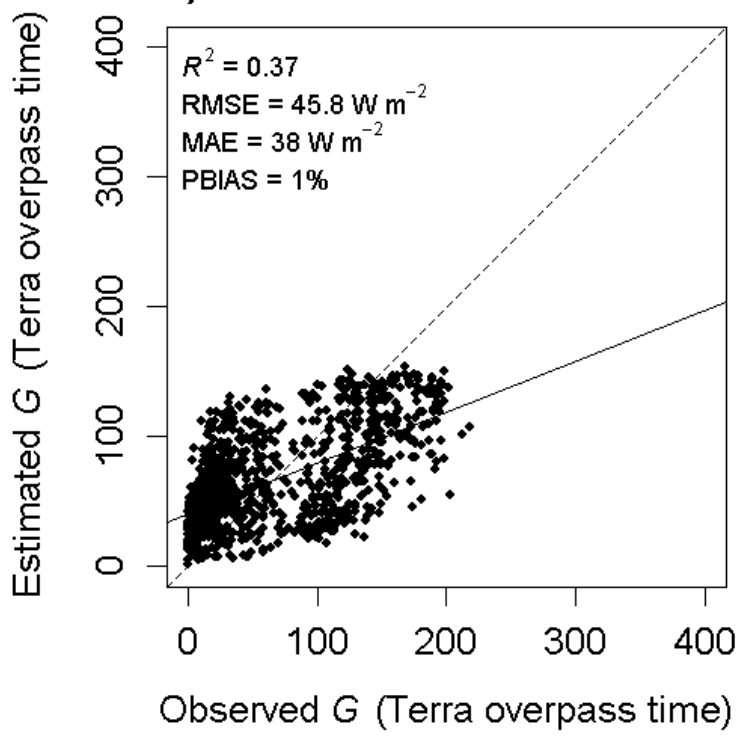

c)

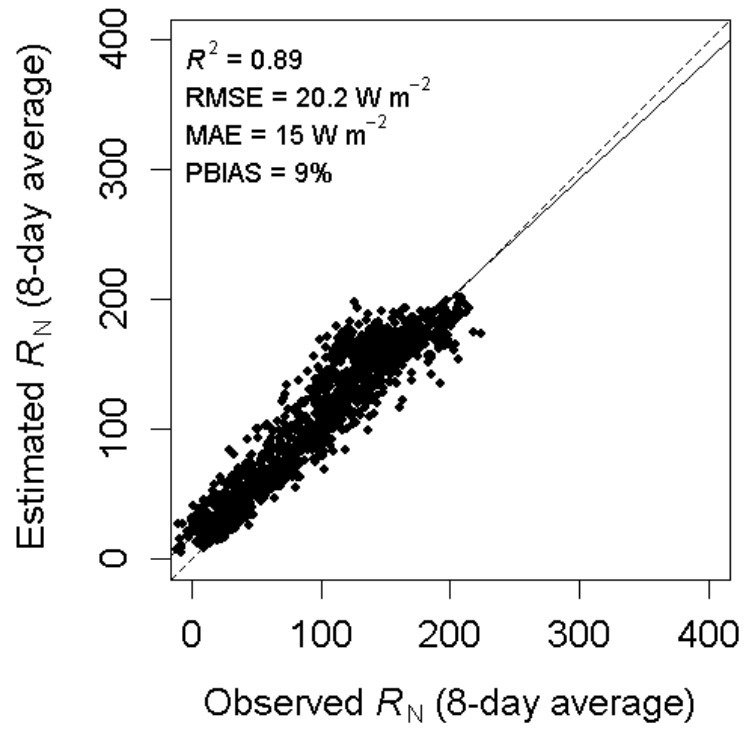

Figure S1: Scatterplots of estimated vs observed (a) $R_{\mathrm{N}}$, (b) $G$, (c) 8-day average daily $R_{\mathrm{N}}$ at the 13 core AmeriFlux sites considered in this study. 

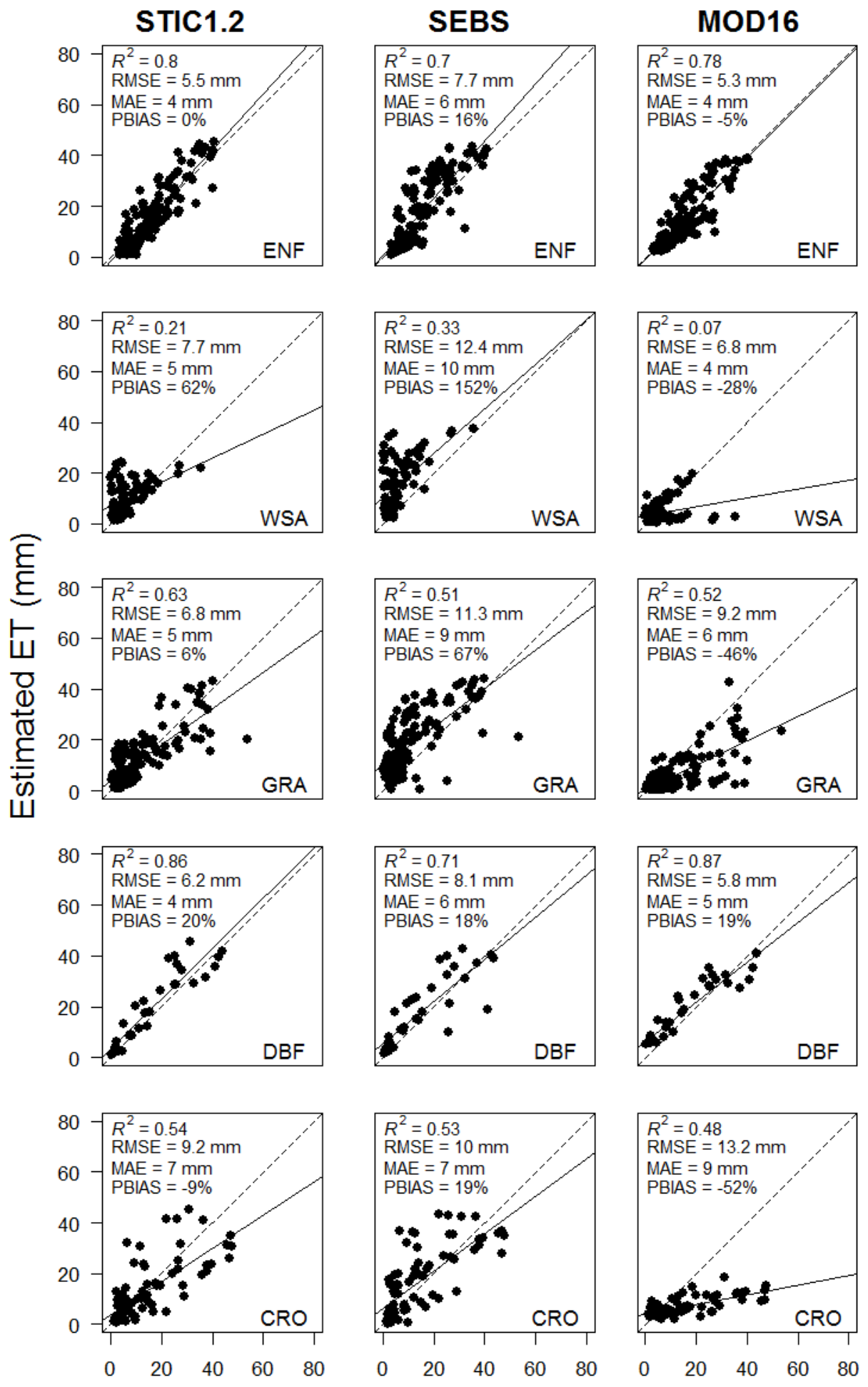

Observed ET (mm)

Figure S2: Evaluation of 8-day cumulative ET from STIC1.2, SEBS, and MOD16 aggregated on different biome types against ET observation at the $\mathbf{1 3}$ core AmeriFlux sites during dry years. 


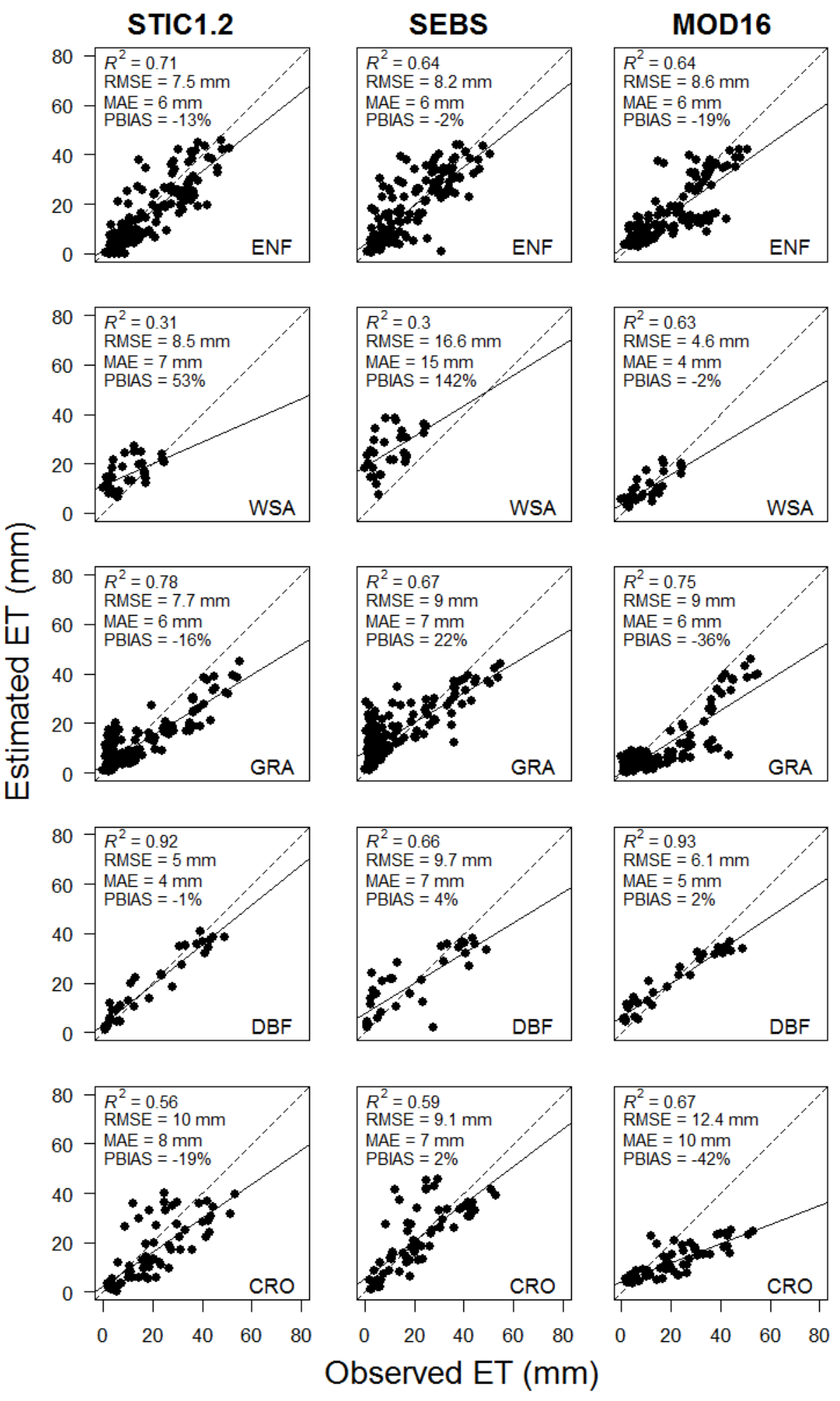

Figure S3: Evaluation of 8-day cumulative ET from STIC1.2, SEBS, and MOD16 aggregated on different biome types against ET observation at the 13 core AmeriFlux sites during wet years. 
STIC1.2
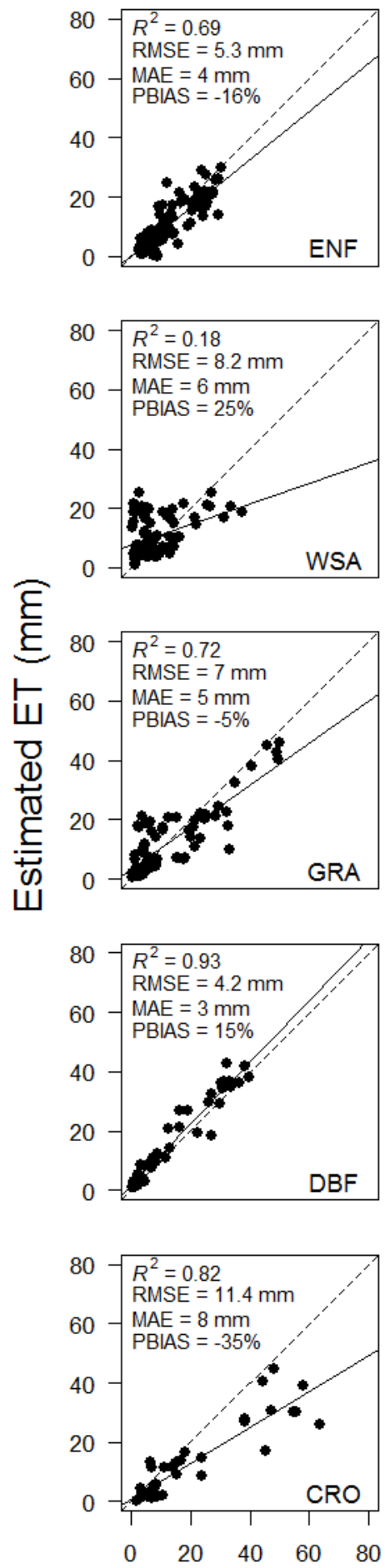
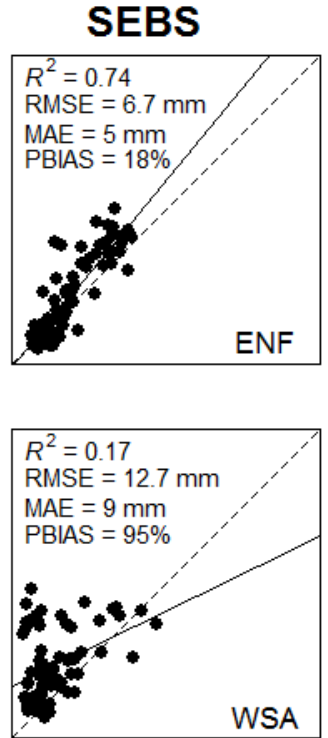

WSA
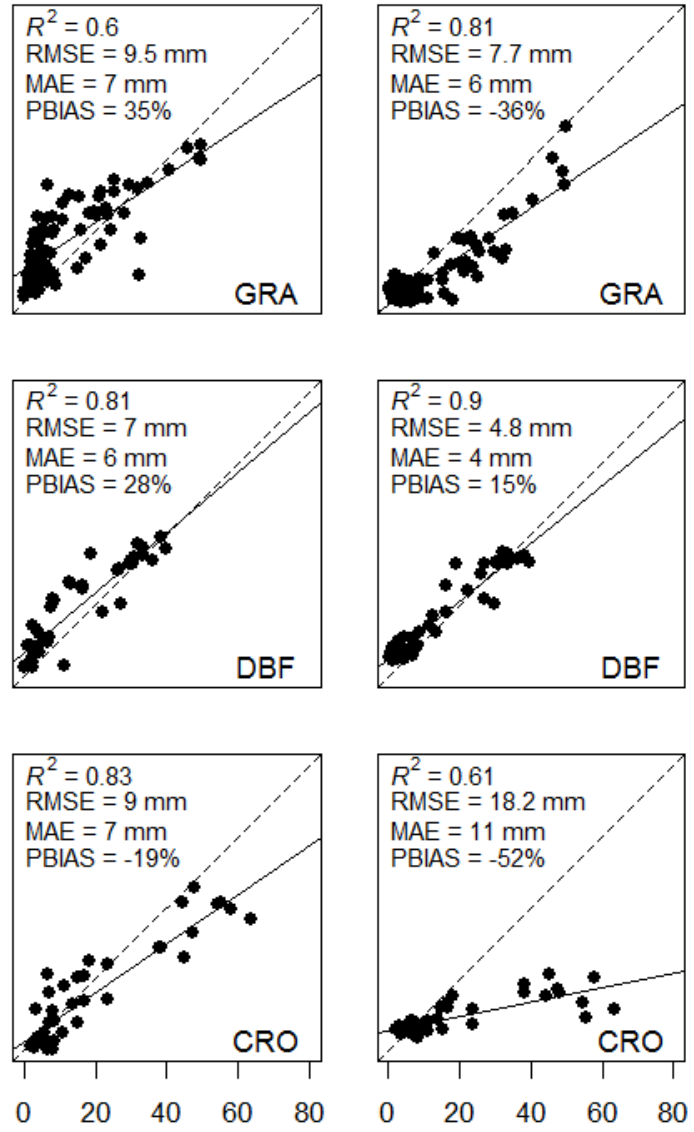

Observed ET (mm)
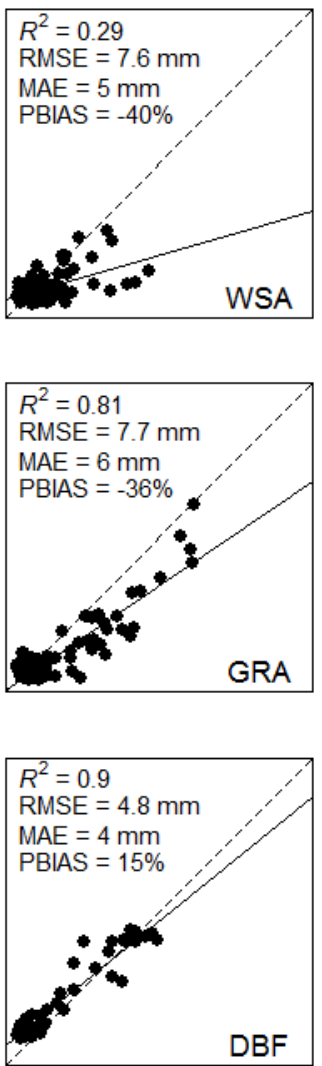

Figure S4: Evaluation of 8-day cumulative ET from STIC1.2, SEBS, and MOD16 aggregated on different biome types against ET observation in the thirteen AmeriFlux sites during normal precipitation year. 

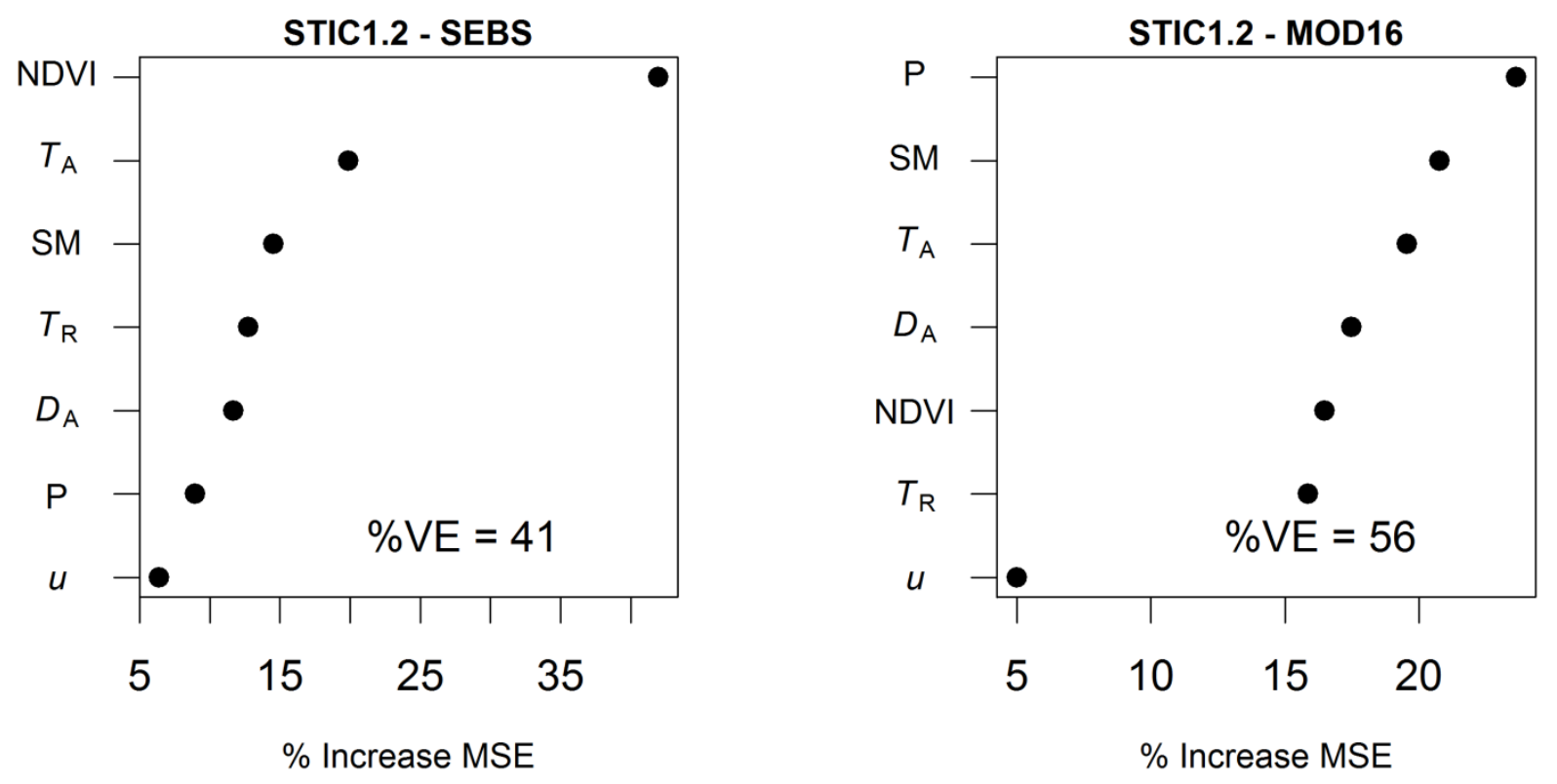

Figure S5: Random forest results for residual difference between 8-day cumulative ET from STIC1.2 and either SEBS or MOD16, when all sites were lumped together. Each plot shows the relative importance of one of seven variables considered in the model, with the most important variable having the largest \% increase in mean squared error (MSE) between the full models and when that variable is permuted. \%VE indicates \% in available variance explained across all trees in the out of bag sample recorded for the full model. 

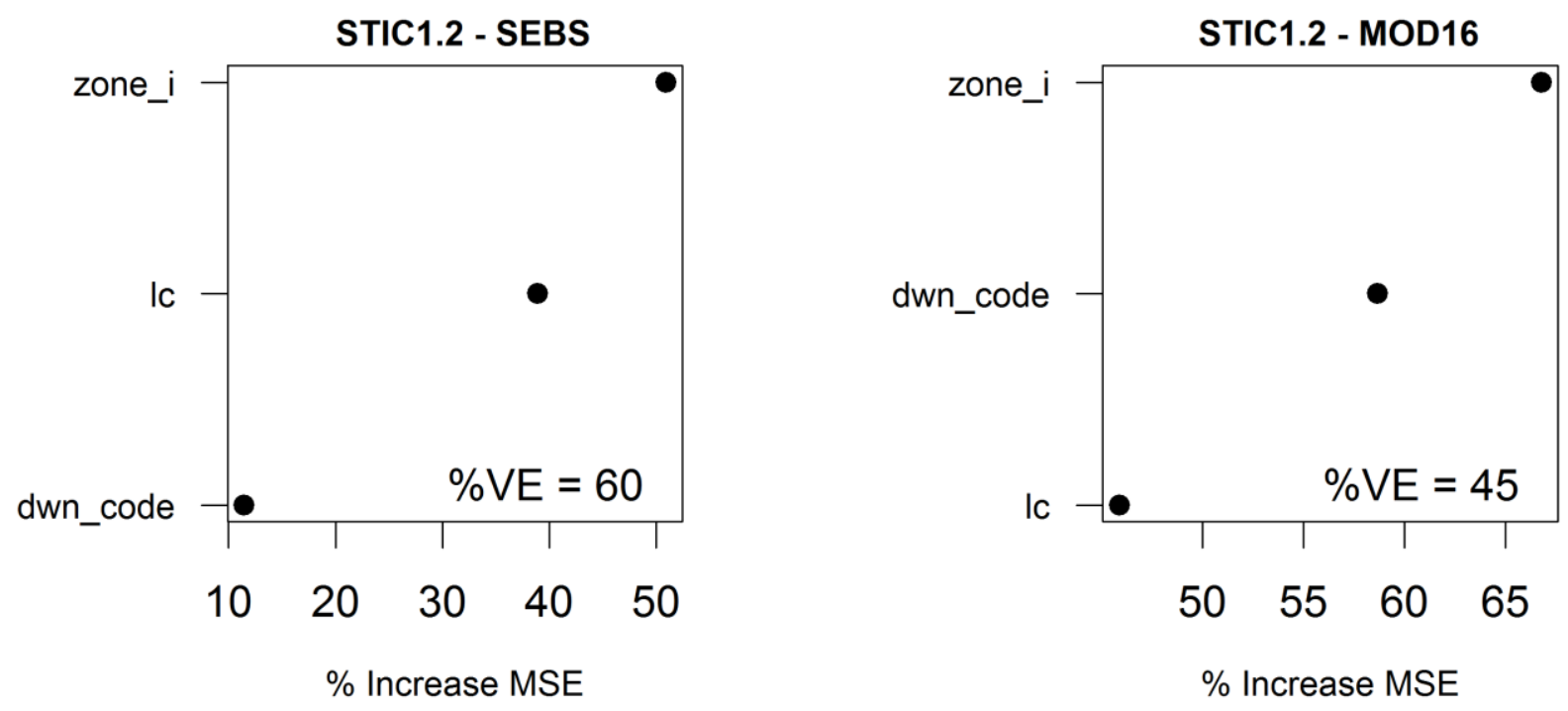

Figure S6: Random forest results for residual difference between annual ET from STIC1.2 and either SEBS or MOD16, when all sites were lumped together. Each plot shows the relative importance of one of three categorical variables considered in the model, with the most important variable having the largest \% increase in mean squared error (MSE) between the full models and when that particular variable is permuted. \%VE indicates \% in available variance explained across all tree in the out of bag sample recorded for the full model. The three categorical variables are for drought conditions [dwn_code $=1($ dry), 2(wet), and 3(normal)], land cover type [lc_year $=$ land cover class], and US zones considered in this study [zone_ $i=$ one of the four zones (W, MW1, MW2, E)]. 